\title{
Correction: Nanophotonics shines light on hyperbolic metamaterials
}

Andreas Aigner, Judith M. Dawes, Stefan A. Maier and Haoran Ren (1)

\section{Correction to: Light: Science \& Applications https://doi.org/10.1038/s41377-021-00688-2, published online 10 January 2022}

Following publication of this article ${ }^{1}$, it was noted that this article contains some errors. The order of Fig. 1c, d was reversed. Figure 1 has been updated in this Correction. The original article has been updated as well.
Published online: 26 January 2022

\section{Reference}

1. Aigner, A. et al. Nanophotonics shines light on hyperbolic metamaterials. Light Sci. Appl. 11, 9 (2022). 


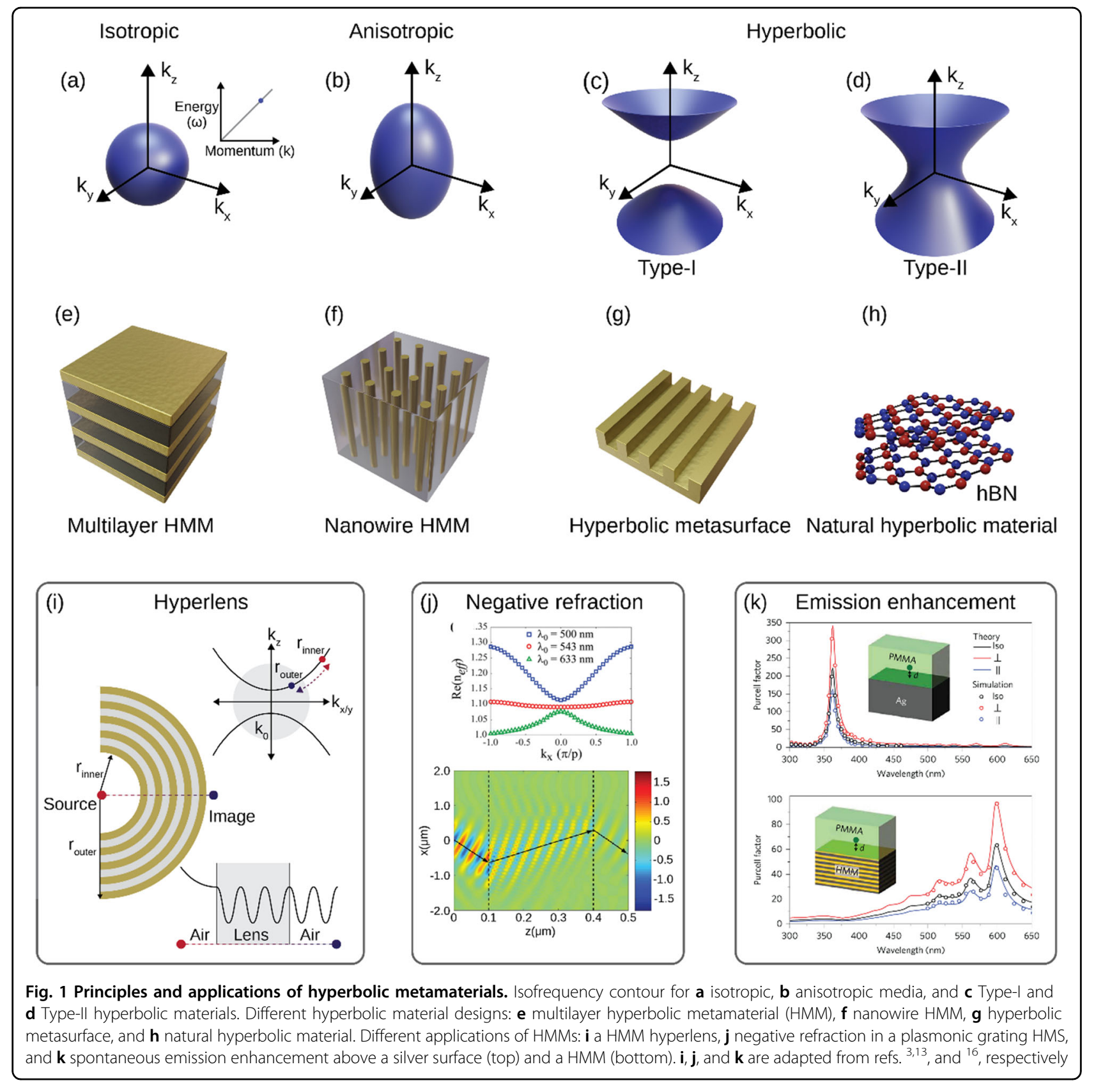

